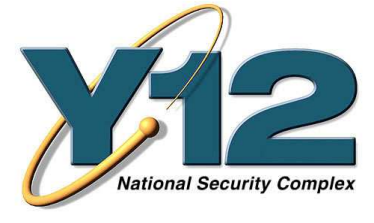

A BWXT/Bechtel Enterprise

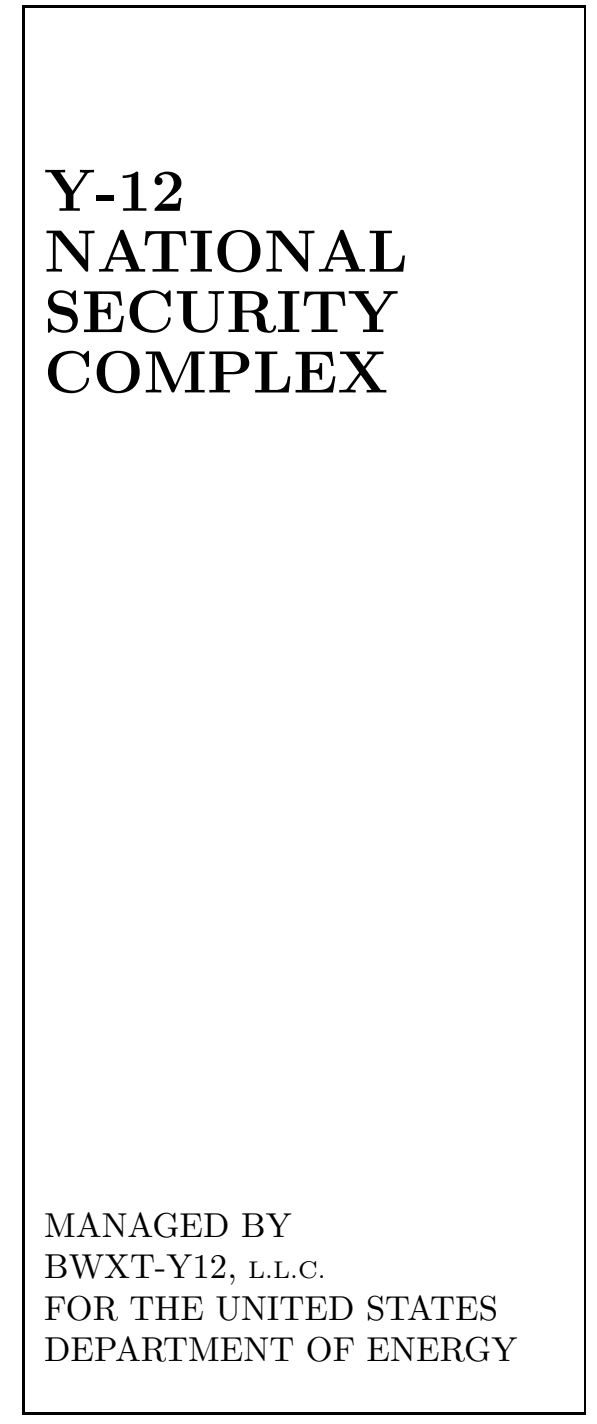

Document No. Y/DK-2107

Improved Background Corrections for Uranium Holdup Measurements

\author{
R. B. Oberer \\ C. A. Gunn \\ L. G. Chiang
}

June 21, 2004

Prepared by the Y-12 National Security Complex

P.O. Box 2009

Oak Ridge, TN 37831-8169

managed by

BWXT-Y12, L.L.C.

for the

U.S. DEPARTMENT OF ENERGY

under contract DE-AC05-00OR22800 


\section{DISCLAIMER}

This report was prepared as an account of work sponsored by an agency of the United States Government. Neither the United States Government nor any agency thereof, nor any of their employees, makes any warranty, express or implied, or assumes any legal liability or responsibility for the accuracy, completeness, or usefulness of any information, apparatus, product, or process disclosed, or represents that its use would not infringe privately owned rights. Reference herein to any specific commercial product, process, or service by trade name, trademark, manufacturer, or otherwise does not necessarily constitute or imply its endorsement, recommendation, or favoring by the United States Government or any agency thereof. The views and opinions of authors expressed herein do not necessarily state or reflect those of the United States Government or any agency thereof.

\section{COPYRIGHT NOTICE}

The submitted manuscript has been authored by a subcontractor of the U. S. Government under contract DE-AC05-00OR-22800. Accordingly, the U. S. Government retains a paid-up, nonexclusive, irrevocable, worldwide license to publish or reproduce the published form of this contribution, prepare derivative works, distribute copies to the public, and perform publicly and display publicly, or allow others to do so, for U. S. Government purposes. 


\title{
Improved Background Corrections for Uranium Holdup Measurements
}

\author{
R. B. Oberer \\ C. A. Gunn \\ L. G. Chiang
}

October 26, 2004

\section{Introduction}

In the original Generalized Geometry Holdup (GGH) model, all holdup deposits were modeled as points, lines, and areas $[1,5]$. Two improvements[4] were recently made to the GGH model and are currently in use at the Y-12 National Security Complex. These two improvements are the finite-source correction $C F_{g}$ and the self-attenuation correction. ${ }^{1}$ The finite-source correction ${ }^{2}$ corrects the average detector response for the width of point and line geometries which in effect, converts points and lines into areas. The result of a holdup measurement of an area deposit is a density-thickness ${ }^{3}$ which is converted to mass by multiplying it by the area of the deposit. From the measured density-thickness, the true density-thickness can be calculated by correcting for the material self-attenuation. Therefore the self-attenuation correction is applied to finite point and line deposits as well as areas.

This report demonstrates that the finite-source and self-attenuation corrections also provide a means to better separate the gamma rays emitted by the material from the gamma rays emitted by background sources for an improved background correction. Currently, the measured background radiation is attenuated for equipment walls in the case of area deposits but not for line and point sources. The measured background radiation is not corrected for attenuation by the uranium material. For all of these cases, the background is overestimated which causes a negative bias in the measurement. The finite-source correction and the selfattenuation correction will allow the correction of the measured background radiation for both the equipment attenuation and material attenuation for area sources as well as point and line sources.

The self-attenuation correction was originally derived from Equation $(1)^{4}$

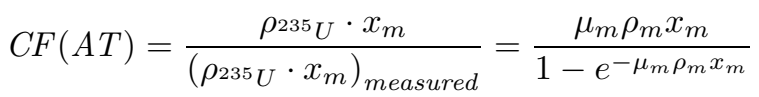

where $\mu_{m}$ is the mass attenuation coefficient of the uranium material $m, \rho_{m}$ is the density of the material, $\rho^{235} U$ is the density of ${ }^{235} U$, and $x_{m}$ is the thickness of the material. By noting that the density of ${ }^{235} U$ in the material is related to the density of the material by the weight fraction, $\rho_{235} U=f_{235} U \cdot \rho_{m}$, Equation (1) can be solved for the true density-thickness of ${ }^{235} U$ as shown in Equation (2) below:

$$
\rho^{235} U \cdot x_{m}=-\frac{f_{235} U}{\mu_{m}} \ln \left[1-\frac{\mu_{m}}{f_{235} U} \cdot\left(\rho_{235} U \cdot x_{m}\right)_{\text {measured }}\right] .
$$

\footnotetext{
${ }^{1}$ Both the finite-source correction and the self-attenuation correction are integrated into HMS4. In HMS3, these corrections are calculated with separate programs, the Geometric Response Correction and Self-Attenuation Correction, Ver 1.0. or in a spreadsheet.

${ }^{2}$ For an improvement to the finite-source correction, see Reference [2].

${ }^{3}$ Density-thickness is sometimes referred to as an areal density.

${ }^{4}$ This equation originates from Equation(6-6) in Reference [3].
} 
Equation (2) is only correct when there is no background radiation. The problem is that the background radiation, corrected for attenuation by the true density-thickness of the material, must be subtracted to arrive at the measured density-thickness of the material, $\left(\rho_{235} U \cdot x_{m}\right)_{\text {measured }}$. Unfortunately, the true densitythickness of the material is not known prior to performing the self-attenuation correction. In practice, for area sources, an equipment attenuated background is subtracted instead. For point and line sources an unattenuated background is subtracted. If both the background and self-attenuation are significant, the background is overstated resulting in a significant underestimate of the true density-thickness.

An example will demonstrate the problem. Suppose we measure uranium-loaded material in a small container with a collimated detector as represented in Figure 1. The container wall is labeled "Equipment, $e$ " in the figure. Notice that the detector measures a count rate $R^{\prime \prime}$ that includes both the background $B_{0}$ and the gamma rays from the uranium material which are attenuated by both the material itself and a layer of equipment. Furthermore, let's assume that the uranium material is $93 \%$ enriched uranium in the form of $U_{3} O_{8}$. Thus, the weight fraction of grams of ${ }^{235} U$ to the grams of $U_{3} O_{8}, f_{235} \mathrm{U}$, is 0.787 . Suppose the area calibration constant for the detector is $K_{a}=2.0392 \times 10^{-4} \mathrm{~g} \cdot \mathrm{s} / \mathrm{cm}^{2}$. The dimensions of the uranium-loaded material layer and the equipment layer are shown in Table 1.

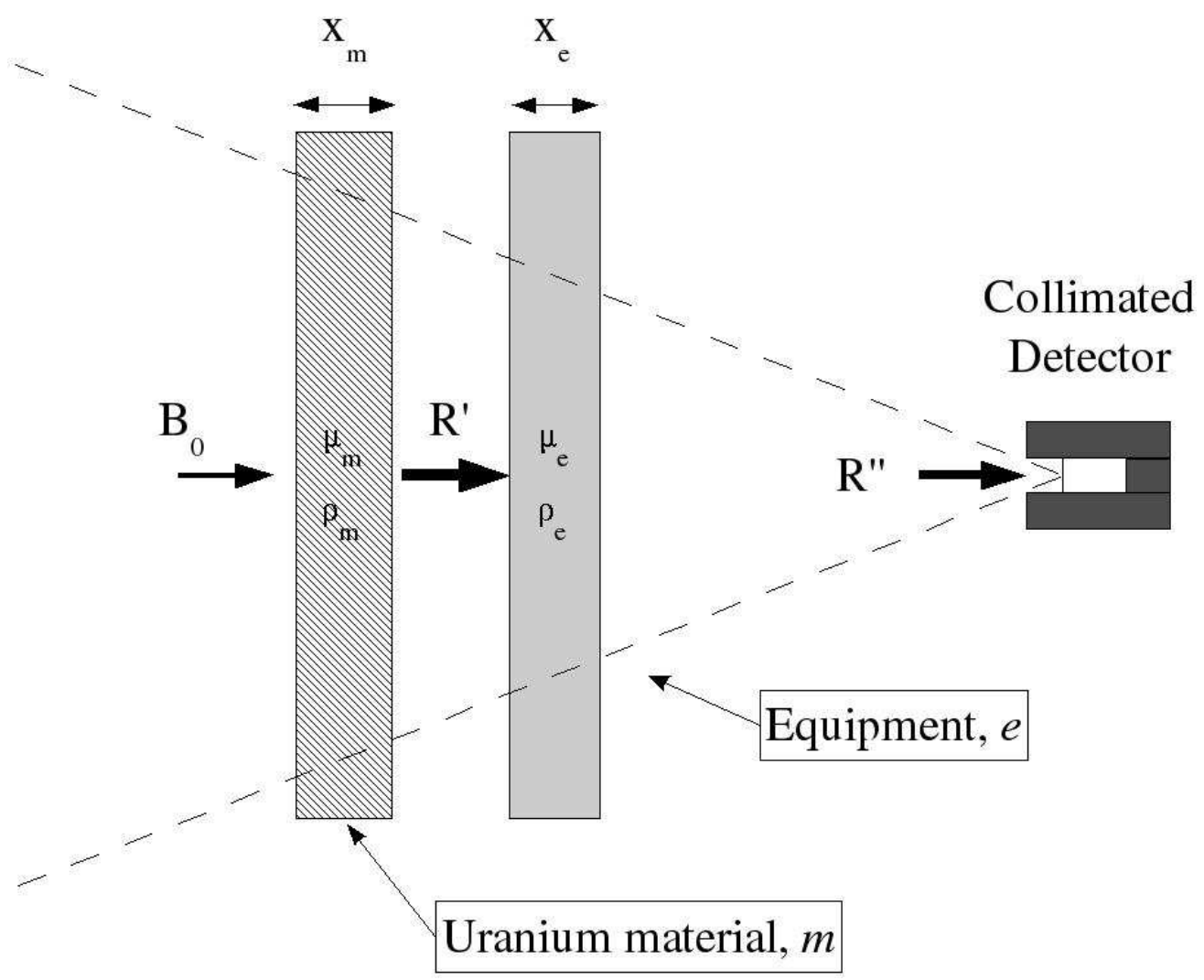

Figure 1: Model of a slab of material measured with a collimated detector. The detector detects a count rate $R^{\prime \prime}$ from both the uranium material and background $186 \mathrm{keV}$ gamma rays attenuated for both the material and equipment. A count rate $R^{\prime}$ can be calculated by correcting for equipment attenuation which is what the detector would have measured if the equipment were not present.

Using the dimensions for the equipment layer, the attenuation correction factor is $C F_{e}=1.25$. Suppose we measure a net count rate from $186 \mathrm{keV}$ gamma rays, $R^{\prime \prime}=1109 \mathrm{cps}$. The net $186 \mathrm{keV}$ count rate from the background is measured, $B_{0}=800 \mathrm{cps}$. The net background-corrected count rate corrected for equipment 
Table 1: Uranium and Equipment Specifications

\begin{tabular}{|c|c|c|c|c|}
\hline Layer & & $\begin{array}{l}\text { thickness } \\
(\mathrm{cm})\end{array}$ & $\begin{array}{l}\text { density } \\
\left(\mathrm{g} / \mathrm{cm}^{3}\right)\end{array}$ & $\begin{array}{l}\text { mass attenuation constant } \\
\qquad\left(\mathrm{cm}^{2} / \mathrm{g}\right)\end{array}$ \\
\hline Material, & $\begin{array}{l}m=U_{3} O_{8} \\
f_{U}=0.787\end{array}$ & $x_{m}=?$ & $\rho_{m}=?$ & $\mu_{m}=1.259$ \\
\hline Equipment, & $e=F e$ & $x_{e}=0.2$ & $\rho_{e}=7.81$ & $\mu_{e}=0.144$ \\
\hline
\end{tabular}

attenuation is therefore $586 \mathrm{cps}$. The measured density-thickness of ${ }^{235} U$ is $0.120 \mathrm{~g} / \mathrm{cm}^{2}$. Applying Equation 2 for self-attenuation correction results in a corrected density-thickness of $0.134 \mathrm{~g} / \mathrm{cm}^{2}$.

Suppose we discover that most of the background is coming from similar containers nearby. To improve the measurement, we have this nearby material moved. Now the background reads $40 \mathrm{cps}$. The material now reads $661 \mathrm{cps}$. The corrected density-thickness of ${ }^{235} U$ is now $0.186 \mathrm{~g} / \mathrm{cm}^{2}$, an increase of almost $40 \%$. The fact is that we are still slightly underestimating the density-thickness of ${ }^{235} \mathrm{U}$. We are subtracting 32 of the 40 cps background when in reality only about 24 cps are actually transmitted through both the material and equipment. We have failed to adjust the background for the attenuation of the uranium material in both cases. The true density-thickness of ${ }^{235} U$ in this example is $0.189 \mathrm{~g} / \mathrm{cm}^{2}$.

\section{Derivation of self-attenuation correction with background sub- traction}

\subsection{Areas deposits}

The solution to this problem is to include the material corrected background in Equation (1). For the slab geometry of an area source as shown in Figure 1 we measure a count rate of $R^{\prime \prime}$ which is related to $R^{\prime}$ by the equipment attenuation factor,

$$
R^{\prime \prime}=R^{\prime} e^{-\mu_{e} \rho_{e} x_{e}} .
$$

In a separate measurement we also measure the unattenuated background, $B_{0}$. Thus, the total count rate before equipment attenuation is

$$
R^{\prime}=\frac{\epsilon A_{s} I}{\mu_{m}}\left(1-e^{-\mu_{m} \rho_{m} x_{m}}\right)+B_{0} e^{-\mu_{m} \rho_{m} x_{m}} .
$$

$A_{s}$ is the area of the slab, $\epsilon$ is the detection efficiency, and $I$ is the specific emission rate of the $186 \mathrm{keV}$ gamma rays emitted from the ${ }^{235} U$ in the uranium material.

The true count rate $R$ which would have been observed without self-attenuation or equipment attenuation is

$$
R=\epsilon A_{s} I \rho_{m} x_{m}
$$

To determine the true density-thickness from the measured density-thickness, the material attenuated background must be subtracted from the measured count rate $R^{\prime}$. Therefore, Equation (1) is rewritten as

$$
\frac{R}{R^{\prime}-B_{0} e^{-\mu_{m} \rho_{m} x_{m}}}=\frac{\mu_{m} \rho_{m} x_{m}}{1-e^{-\mu_{m} \rho_{m} x_{m}}} .
$$

This equation is then solved to arrive at the true density-thickness of the material, uranium or ${ }^{235} U$ isotope. When doing this, it is important to keep the components of the attenuation straight. The mass attenuation coefficient of the material is calculated by observing that

$$
\mu_{m} \rho_{m} x_{m}=\sum_{i} \mu_{i} \rho_{i} x_{m}=\sum_{i} \mu_{i} f_{i} \rho_{m} x_{m}
$$


where $f_{i}$ is the mass fraction for the ith component. Therefore

$$
\mu_{m}=\sum_{i} \mu_{i} f_{i}
$$

In the case of materials such as $U_{3} O_{8}$ or uranium metal, the mass fractions are constant and known. For uranium solutions however, the mass fractions are generally the unknown value to be determined from NDA.

The density-thickness of ${ }^{235} U$ is determined from the unknown, true count rate $R$ by

$$
\rho_{235} U \cdot x_{m}=K_{a} R .
$$

It can be converted to a density-thickness of uranium by dividing by the fraction of ${ }^{235} U$ to $U$. It can also be converted to a density-thickness of material by dividing by $f^{235} \mathrm{U}$, the fraction of ${ }^{235} U$ to total material.

To solve Equation (6) we can convert to the density-thickness of the material

$$
\rho_{m} x_{m}=\frac{K_{a} R}{f_{235} U}
$$

and solve for the true count rate $R$ in terms of the transmission $T_{m}=e^{-\mu_{m} \rho_{m} x_{m}}$

$$
R=-\frac{f_{235} U \ln \left(T_{m}\right)}{\mu_{m} K_{a}}
$$

where $\ln \left(T_{m}\right)=-\mu_{m} \rho_{m} x_{m}$. Equation (6) is then rewritten in terms of the transmission factor $T_{m}$ and the true count rate $R$ which results in

$$
-\frac{f_{235} U \ln \left(T_{m}\right)}{\mu_{m} K_{a}\left(R^{\prime}-B_{0} T_{m}\right)}=\frac{-\ln \left(T_{m}\right)}{1-T_{m}} .
$$

The calculated transmission can now be solved in several steps:

$$
\begin{aligned}
\frac{f_{235} U}{\mu_{m} K_{a}}\left(1-T_{m}\right) & =R^{\prime}-B_{0} T_{m} \\
T_{m}\left(B_{0}-\frac{f_{235} U}{\mu_{m} K_{a}}\right) & =R^{\prime}-\frac{f_{235} U}{\mu_{m} K_{a}} \\
T_{m} & =\frac{\mu_{m} K_{a} R^{\prime}-f_{235} U}{\mu_{m} K_{a} B_{0}-f_{235} U} .
\end{aligned}
$$

By equating this calculated transmission to the original definition, $T_{m}=e^{-\mu_{m} \rho_{m} x_{m}}$, the density-thickness of the material is solved:

$$
\rho_{m} x_{m}=-\frac{1}{\mu_{m}} \ln \left(T_{m}\right)=-\frac{1}{\mu_{m}} \ln \left(\frac{\mu_{m} K_{a} R^{\prime}-f_{235} U}{\mu_{m} K_{a} B_{0}-f_{235} U}\right) .
$$

The true density-thickness of uranium and the isotope ${ }^{235} \mathrm{U}$ are

$$
\begin{aligned}
\rho_{U} \cdot x_{m} & =f_{U} \cdot \rho_{m} x_{m}=-\frac{f_{U}}{\mu_{m}} \ln \left(T_{m}\right), \text { and } \\
\rho^{235} U \cdot x_{m} & =f_{235} \cdot \rho_{m} x_{m}=-\frac{f_{235}}{\mu_{m}} \ln \left(T_{m}\right) \text { respectively }
\end{aligned}
$$


Equation (8) is equivalent to Equation (2) when there is no background. Setting the background to zero in Equation (7), the transmission becomes

$$
T_{m}=1-\frac{\mu_{m} K_{a} R^{\prime}}{f^{235} U} .
$$

Since the measured density-thickness without the self-attenuation correction is $\left(\rho_{235} U \cdot x_{m}\right)_{\text {measured }}=K_{a} R^{\prime}$, the transmission can be written as

$$
T_{m}=1-\frac{\mu_{m}}{f_{235} U}\left(\rho_{235} U \cdot x_{m}\right)_{\text {measured }}
$$

Now substituting back into Equation (10) we have

$$
\rho_{235} U \cdot x_{m}=-\frac{f_{235} U}{\mu_{m}} \ln \left(1-\frac{\mu_{m}}{f_{235} U}\left(\rho^{235} U \cdot x_{m}\right)_{\text {measured }}\right) .
$$

\subsection{Extension to point and line sources}

In the case of finite point and line sources, some of the measured background will be detected without equipment and material attenuation. The current holdup measurement practice is to assume that all of the background is detected without attenuation for point and line sources. For more accuracy and consistency with area sources, the background can be divided into three parts as shown in Figure 2: a portion that is not attenuated by either the material or equipment which requires no corrections, a portion that is attenuated by the equipment only which will require an equipment attenuation correction and a portion that is attenuated by both the material and equipment which will require attenuation corrections for both.

These portions of the background will be divided up by the effective areas of the detector. The effective area is the detector-response-weighted region of the detector field of view. The effective areas of a finite point or line source $A_{w m},{ }^{5}$ of the equipment $w_{w e}$ and of an area source $A$ are defined as follows:

$$
\begin{gathered}
A=\frac{1}{C_{0}} \sum_{i=0}^{N} a_{i} C_{i} \\
A_{w e}=\frac{1}{C_{0}} \sum_{i=0}^{N} a_{w e_{i}} C_{i}
\end{gathered}
$$

and

$$
A_{w m}=\frac{1}{C_{0}} \sum_{i=0}^{N} a_{w m_{i}} C_{i}
$$

where $a_{w m_{i}}$ is the area of the intersection of the finite point or line deposit with annulus $i$ of the radial response as defined in Reference [2] and $a_{w e_{i}}$ is the area of the intersection of the equipment with annulus $i$ of the radial response.

The part of the background that is attenuated by both the material and equipment will be proportional to the effective area of the point or line source, $A_{w m}$, divided by the effective area of an area source, $A$. The part

\footnotetext{
${ }^{5}$ See Reference [2] for a definition of the effective area, $A_{w m}$. In that reference this area is referred to as $A_{w}$
} 


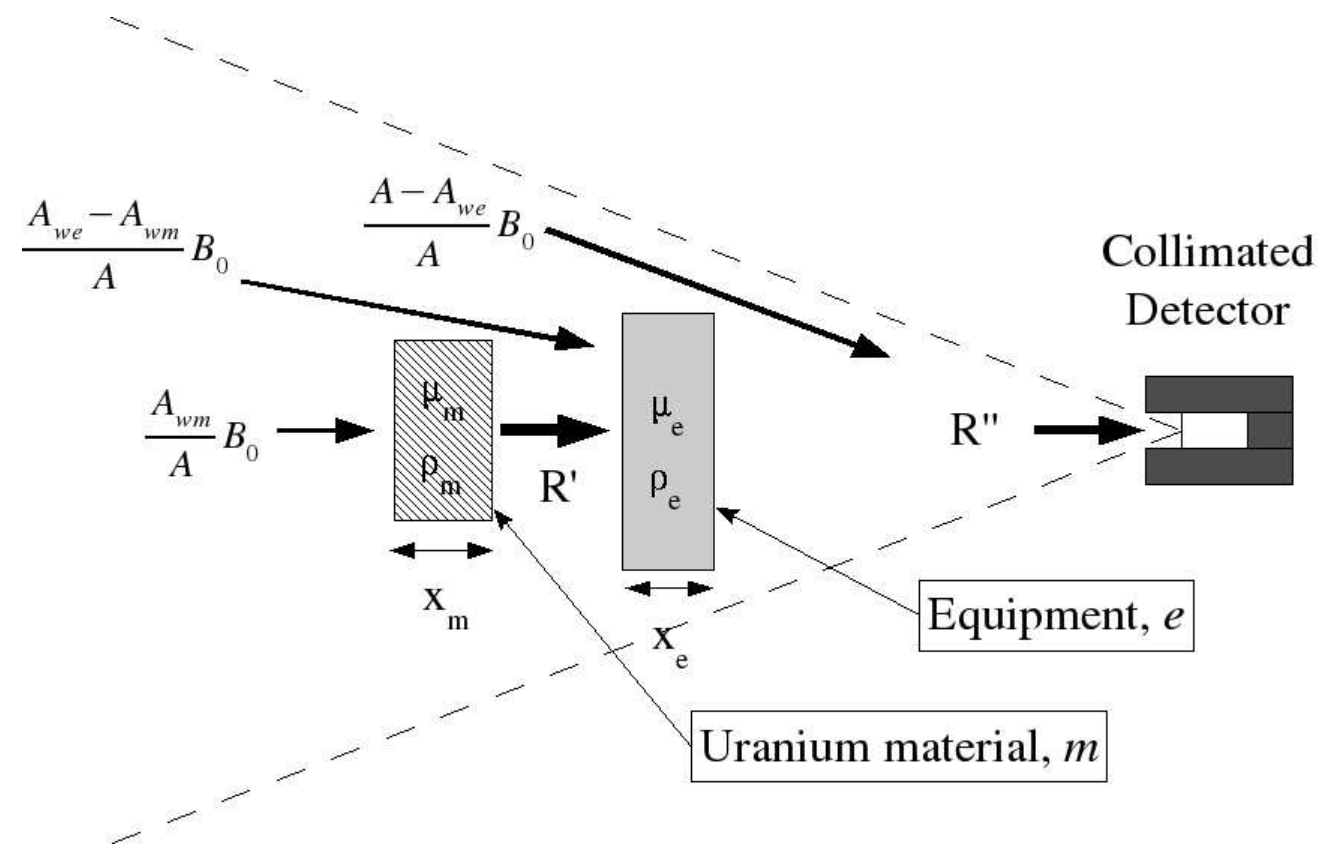

Figure 2: Model of a finite point or line deposit measured with a collimated detector. The detector detects a count rate $R^{\prime \prime}$ from both the uranium material and background $186 \mathrm{keV}$ gamma rays. Some of this background is not attenuated. Some of the background is attenuated by only the equipment and some is attenuated by both the material and equipment.

of the background that is attenuated by only the equipment will be $\frac{A_{w e}-A_{w m}}{A}$. The unattenuated portion of the background is proportional to $\frac{A-A_{w e}}{A}$. The measured response in terms of $R^{\prime}$ and the background $B_{0}$ is

$$
R^{\prime \prime}=\left(R^{\prime}+\frac{A_{w e}-A_{w m}}{A} B_{0}\right) e^{-\mu_{e} \rho_{e} x_{e}}+\frac{A-A_{w e}}{A} B_{0} .
$$

The measured response can be corrected for two of the components of background and equipment attenuation to arrive at $R^{\prime}$ :

$$
R^{\prime}=\left(R^{\prime \prime}-\frac{A-A_{w e}}{A} B_{0}\right) e^{\mu_{e} \rho_{e} x_{e}}-\frac{A_{w e}-A_{w m}}{A} B_{0} .
$$

where

$$
R^{\prime}=\frac{\epsilon A I}{\mu_{m}}\left(1-e^{-\mu_{m} \rho_{m} x_{m}}\right)+\frac{A_{w m}}{A} B_{0} e^{-\mu_{m} \rho_{m} x_{m}}
$$

is similar to Equation (4) except that for point and line deposits only a fraction of the background is attenuated by the material. Now that we have $R^{\prime}$ for point and line sources, we can again take the ratio as in Equation (1) and Equation (6)

$$
\frac{R}{R^{\prime}-\frac{A_{w m}}{A} B_{0} e^{-\left(\mu_{m} \rho_{m} x_{m}\right)}}=\frac{\mu_{m} \rho_{m} x_{m}}{1-e^{-\mu_{m} \rho_{m} x_{m}}} .
$$

The transmission factor $T_{m}$ differs between a point and line source because the density-thickness of the deposit in terms of the true count rate $R$ differs. For a point source, the density-thickness is

$$
\rho_{m} x_{m}=\frac{4 K_{p} C F_{g} R r^{2}}{\pi w^{2} f_{235} U} .
$$


Again, this equation can be rewritten by making the following substitutions for the transmission factor $T_{m}$ and the count rate for a point source, $R$ :

$$
T_{m}=e^{-\mu_{m} \rho_{m} x_{m}}
$$

and

This gives us

$$
R=\frac{\pi w^{2} f_{235} \ln \left(T_{m}\right)}{4 \mu_{m} r^{2} K_{p} C F_{g}}
$$

$$
\frac{\pi w^{2} f_{235} U \ln \left(T_{m}\right)}{4 \mu_{m} r^{2} K_{p} C F_{g}}=\frac{\ln \left(T_{m}\right)}{1-T_{m}}\left(R^{\prime}-\frac{A_{w m}}{A} B_{0} T_{m}\right) .
$$

The transmission factor $T_{m}$ can be solved using the following sequence of steps:

$$
\begin{gathered}
\frac{\pi w^{2} f_{235} U}{4 \mu_{m} r^{2} K_{p} C F_{g}}\left(1-T_{m}\right)=R^{\prime}-\frac{A_{w m}}{A} B_{0} T_{m} \\
T_{m}\left(\frac{A_{w m}}{A} B_{0}-\frac{\pi w^{2} f_{235} U}{4 \mu_{m} r^{2} K_{p} C F_{g}}\right)=R^{\prime}-\frac{\pi w^{2} f_{235} U}{4 \mu_{m} r^{2} K_{p} C F_{g}} \\
T_{m}=\frac{4 \mu_{m} r^{2} K_{p} C F_{g} R^{\prime}-\pi w^{2} f_{235} U}{4 \mu_{m} r^{2} K_{p} C F_{g} \frac{A_{w m}}{A} B_{0}-\pi w^{2} f_{235} U}
\end{gathered}
$$

For a line source the density-thickness is

$$
\rho_{m} x_{m}=\frac{K_{l} C F_{g} R r}{w f_{235} U}
$$

resulting in

$$
R=\frac{w f_{235} U \ln \left(T_{m}\right)}{\mu_{m} r K_{l} C F_{g}} .
$$

The transmission factor is then solved as before:

$$
T_{m}=\frac{\mu_{m} r K_{l} C F_{g} R^{\prime}-w f_{235} U}{\mu_{m} r K_{l} C F_{g} \frac{A_{w m}}{A} B_{0}-w f_{235} U} .
$$

Once the transmission factor $T_{m}$ is determined for a point, line, or area source, the density-thickness of the material is calculated using Equation (8)

$$
\rho_{m} x_{m}=-\frac{1}{\mu_{m}} \ln \left(T_{m}\right)
$$

and the true density-thickness of uranium and the ${ }^{235} U$ isotope are $\rho_{U} x_{m}=f_{U} \cdot \rho_{m} x_{m}$ and $\rho_{235} U \cdot x_{m}=$ $f_{235} U \cdot \rho_{m} x_{m}$ respectively.

\section{Conclusion}

As shown in the previous section, it is a simple matter to solve Equation (6) and Equation (13) for the true density-thickness of the material in terms of the transmission factor $T_{m}$. The transmission factor $T_{m}$ is calculated from the holdup measurement using the formulas summarized in Table 2 for point, line, and area sources. A separate transmission measurement is not required. Once $T_{m}$ is determined, the density-thickness of the material is calculated from

$$
\rho_{m} x_{m}=-\frac{1}{\mu_{m}} \ln \left(T_{m}\right) .
$$


The true density-thickness of uranium and the isotope ${ }^{235} U$ are

$$
\begin{aligned}
\rho_{U} \cdot x_{m} & =f_{U} \cdot \rho_{m} x_{m}=-\frac{f_{U}}{\mu_{m}} \ln \left(T_{m}\right), \text { and } \\
\rho_{235} \cdot x_{m} & =f_{235} \cdot \rho_{m} x_{m}=-\frac{f_{235}}{\mu_{m}} \ln \left(T_{m}\right) \text { respectively. }
\end{aligned}
$$

\begin{tabular}{|c|c|}
\hline Geometry of deposit & Transmission Factor, $T_{m}$ \\
\hline Point & $\frac{4 \mu_{m} r^{2} K_{p} C F_{g} R^{\prime}-\pi w^{2} f_{235_{U}}}{4 \mu_{m} r^{2} K_{p} C F_{g} \frac{A_{w m}}{A} B_{0}-\pi w^{2} f_{235_{U}}}$ \\
\hline Line & $\frac{\mu_{m} r K_{l} C F_{g} R^{\prime}-w f_{235_{U}}}{\mu_{m} r K_{l} C F_{g} \frac{A_{w m}}{A} B_{0}-w f_{235_{U}}}$ \\
\hline Area & $\frac{\mu_{m} K_{a} R^{\prime}-f_{235_{U}}}{\mu_{m} K_{a} B_{0}-f_{235_{U}}}$ \\
\hline $\begin{array}{l}K_{p}, K_{l}, K_{w}: \text { point, li } \\
R^{\prime}: \text { measured count } \\
r: \text { distance from fron } \\
\mu_{m}: \text { mass attenuatio } \\
f_{235}: \text { weight fractiol } \\
C F_{g}: \text { finite-source ge } \\
A: \text { effective area for } \\
A_{w m}: \text { effective area } f \\
w: \text { width of a point } c \\
B_{0}: \text { background cour }\end{array}$ & $\begin{array}{l}\text { and area calibration factors } \\
\text { te, corrected for unattenuated background and equipment attenuation } \\
\text { face of detector crystal to the material } \\
\text { constant of uranium material } \\
\text { of }{ }^{235} U \text { to material } \\
\text { metry correction factor } \\
\text { area source } \\
\text { a finite point or line source of width } w \\
\text { line source } \\
\text { rate }\end{array}$ \\
\hline
\end{tabular}

Table 2: Transmission Factor, $T_{m}$, for point, line, and area sources.

The steps in applying the improved background subtraction are as follows:

1. The total count rate from the deposit through the equipment $R^{\prime \prime}$ and the unattenuated background without the equipment $B_{0}$ are measured as usual. The geometry of the measurement is illustrated in Figure 1.

2. For finite point and line sources, the unattenuated portion of the background $\frac{A-A_{w e}}{A} B_{0}$ is subtracted from $R^{\prime \prime}$.

3. The total count rate $R^{\prime \prime}$, with unattenuated background subtracted, is corrected for equipment attenuation.

4. For point and line sources the portion of the background attenuated by only the equipment $\frac{A_{w e}-A_{w m}}{A} B_{0}$ is subtracted to arrive at $R^{\prime}$.

5. The transmission factor, $T_{m}$ is calculated from $R^{\prime}$ and $B_{0}$ using the equations summarized in Table 2 .

6. From the transmission factor $T_{m}$ and Equation (18), the true density-thickness of ${ }^{235} U$ is calculated.

7. The mass is then finally determined by multiplying the true density-thickness by the area of the finite point, line or area deposit. 
Referring back to Figure 1 and the example problem, $R^{\prime \prime}$ is corrected for equipment attenuation by multiplying by $e^{+\mu_{e} \rho_{e} x_{e}}$ to arrive at $R^{\prime}$. In the example problem $R^{\prime \prime}=1109 \mathrm{cps}$. Therefore $R^{\prime}$ is $1391 \mathrm{cps}$. Applying the equation for an area from Table 2 we determine $T_{m}=0.7388$. From Equation (18) we determine that the true density-thickness of ${ }^{235} U$ is $0.189 \mathrm{~cm}^{2}$.

\section{References}

[1] R. H. Auguston and T. D. Reilly. Fundamentals of Passive Nondestructive Assay of Fissionable Material, chapter 5, pages 45-48. Number LA-5651-M. Los Alamos National Laboratory, September 1974.

[2] C. A. Gunn, R. B. Oberer, L. G. Chiang, and R. N. Ceo. A generalized finite source calibration factor: A natural improvement to the finite source correction factor for uranium holdup measurements. Technical Report Y/DX-2525, Y-12 National Security Complex, January 2003.

[3] D. Reilly, N. Ensslin, H. Smith, Jr., and S. Kreiner. Passive Nondestructive Assay of Nuclear Materials, chapter 6. Number NUREG/CR-5550. United States Nuclear Regulatory Commission, March 1991.

[4] P. A. Russo, T. R. Wenz, S. E. Smith, and J. F. Harris. Acheiving higher accuracy in the gammaray spectroscopic assay of holdup. Technical Report LA-13699-MS, Los Alamos National Laboratory, September 2000.

[5] J. K. Sprinkle, Jr., R. Cole, M. L. Collins, S-T Hsue, P. A. Russo, R. Siebelist, H. A. Smith, Jr., R. N. Ceo, and S. E. Smith. Low-resolution gamma-ray measurements of process holdup. Technical Report LA-UR-96-3482, Los Alamos National Laboratory, October 1996. 